Please do not remove this page

RMIT

UNIVERSITY

\title{
Maximum Likelihood Indoor Localization of a WiFi Radio Transmitter with Structural Knowledge
}

Sun, Shuai; Wang, Xuezhi; Moran, Bill; Hourani, Akram; Rowe, Wayne

https://researchrepository.rmit.edu.au/esploro/outputs/9921863269701341/filesAndLinks?institution=61RMIT_INST\&index=null

Sun, S., Wang, X., Moran, B., Hourani, A., \& Rowe, W. (2017). Maximum Likelihood Indoor Localization of a WiFi Radio Transmitter with Structural Knowledge. Proceedings of the 9th International Conference on Signal Processing Systems 2017, 27-31. https://doi.org/10.1145/3163080.3163090

Document Version: Accepted Manuscript

Published Version: https://doi.org/10.1145/3163080.3163090

Repository homepage: https://researchrepository.rmit.edu.au

(C) 2017 Association for Computing Machinery

Downloaded On 2023/04/26 19:38:54 +1000 


\title{
Maximum Likelihood Indoor Localization of a WiFi Radio Transmitter with Structural Knowledge
}

\author{
Shuai Sun, Xuezhi Wang, Bill Moran, Akram Al-Hourani, Wayne Rowne \\ RMIT university, Melbourne, Australia \\ s3583925@student.rmit.edu.au
}

\begin{abstract}
In this paper, we present a method for estimating the location of a WiFi transmitter by a receiver using the radio resource and knowledge of the indoor room structure. We derive a three-ray path propagation model for the received radio signal in a known indoor environment. We show that the position of the transmitter could be localized using the received radio signal measurements. The likelihood under this model exhibits multiple local peaks when only few frequencies are used, which leads to the location ambiguities under the Maximum Likelihood criterion. We observed in simulation that the ambiguous locations under the MLE vary with the WiFi radio frequency used but the ground truth location is always presented as a peak. Therefore, we use multiple $\mathrm{WiFi}$ frequency bands to resolve the localization ambiguity. A subspace based method is applied in combination with MLE utilizing the same measurements to improve localization efficiency. Simulation using commercial ray tracing software presents promising result.
\end{abstract}

\section{Keywords}

Maximum Likelihood, location ambiguity, WiFi frequency, subspace

\section{INTRODUCTION}

WiFi-based indoor localization has drawn increasing attention in recent years, due to its low cost, ease of implementation and pervasive practical application. Traditional WiFi-based localization methods typically resort to signal parameters such as time of flight based approach for range estimation and then localize the target of interest by making use of multiple access points and triangulation technique [1], or utilize powerful receiver structure such as antenna array for angle of arrival identification and angulation. However, the accuracy and stability of the aforementioned methods usually are affected by multipath interference in indoor environment. On the other hand, even though multipath fading effects can lead to non-neglectable signal variance, i.e.,

Permission to make digital or hard copies of all or part of this work for personal or classroom use is granted without fee provided that copies are not made or distributed for profit or commercial advantage and that copies bear this notice and the full citation on the first page. Copyrights for components of this work owned by others than ACM must be honored. Abstracting with credit is permitted. To copy otherwise, or republish, to post on servers or to redistribute to lists, requires prior specific permission and/or a fee. Request permissions from permissions@ acm.org.

WOODSTOCK'97 El Paso, Texas USA

(C) 2017 ACM. ISBN 123-4567-24-567/08/06 .. \$15.00

DOI: $10.475 / 123 \_4$ constructive and destructive fading, with respect to different operation frequencies and transceiver locations [2], this channel frequency selective fading property may turn out to be helpful in providing an unique mapping for localization purpose.

As WiFi can operate at both $2.4 \mathrm{GHZ}$ and $5 \mathrm{GHZ}$ band, we can exploit its multipath interference and frequency selective fading property to localize the $\mathrm{WiFi}$ transmitter. We consider a WiFi based localization scenario which involves only one radio transmitter and receiver pair but with known room structure and in the presence of direct path signal. A three-ray path propagation model is derived and a Maximum Likelihood estimation (MLE) based approach is proposed to estimate the transmitter location using the available received WiFi signal, which is a complex number in general. To remove location ambiguity, measurements across all the available WiFi frequency bands may be utilized. The use of multiple bands for localization has appeared in recent literature. For example, in [3], WiFi signals at $2.4 \mathrm{GHz}$ and $5 \mathrm{GHz}$ spanning almost one $\mathrm{GHz}$ of bandwidth with a total number of $35 \mathrm{WiFi}$ center frequency bands are used for time of flight estimation. As indicated in [4], the multiple bands WiFi measurements (complex number) can be conveniently extracted from Channel State Information (CSI) in Intel 5300 cards.

This paper is organized as follows. Following the Introduction, we first formulate the receive signal data model in Section II. Then we describe the location ambiguity issue in MLE due to multipath signal interference and briefly explain the idea of addressing ambiguity problem by utilizing multiple WiFi frequency bands in Section III. In Section IV, based on the measured multiple frequency signal, MLE is derived, together with a subspace based method to improve estimation efficiency. In Section V, simulation of MLE localization result using commercial software for scene setting and measurement generation is demonstrated. Finally, conclusions are given in Section VI.

\section{THE DATA MODEL}

Consider the problem of localizing a radio transmitter in a two dimensional space with known room structure and a fixed receiver whose location is given, as shown in Fig. 1. A radio frequency signal is transmitted by the transmitter and multiple copies of the same signal with different time delay and attenuation is superpositioned at the receiver due to reflection. In order to account for this multipath interference as well as reduce complexity, reflected signals that only bounce less than two times are taken as effective components 
in the receiving signal, owing to their relatively significant decaying of amplitude during reflection, while the rest of which are regarded as additional noise.

According to [5], the channel response for frequency $f_{i}$ given the transmitter location $\varphi=(x, y)$ can be modeled as

$$
h_{i}(\varphi)=\sum_{p=0}^{P} a_{p}(\varphi) \exp ^{-j 2 \pi f_{i} \tau_{p}(\varphi)}, \quad i=1,2, \cdots N
$$

where $a_{p}(\varphi)$ and $\tau_{p}(\varphi)$ are the amplitude and time delay for the $p^{\text {th }}$ path, with $p=0$ representing the direct path. Recall that $h_{i}(\varphi)$ is provided as CSI in some available commercial chips, based on the 802.11 standard, such as Intel 5300 NIC [4].

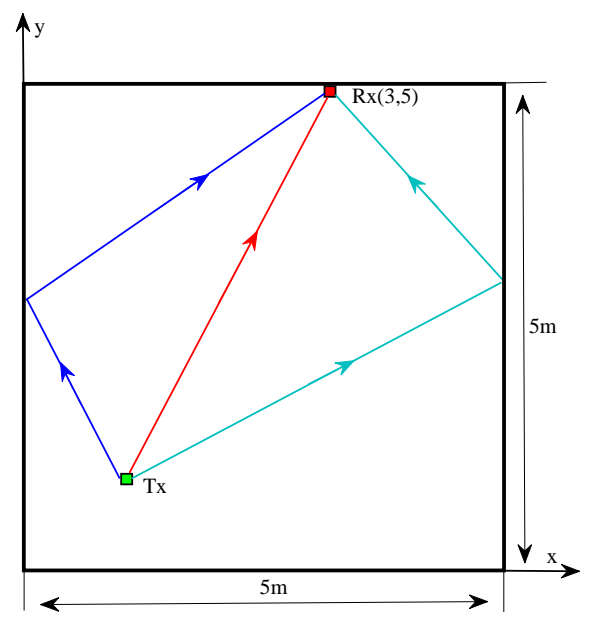

Figure 1: Considered three ray propagation path model: a fixed receiver ( $\mathrm{Rx}$ ) whose location is known receives three components of superpositioned sinusoids emitted by a radio transmitter ( $T x$ ) with unknown location in a given $5 m \times 5 m$ room. Each signal path undergoes different attenuations and time delays.

As the value of $a_{p}$ and $\tau_{p}$ are both functions of the transmitter location $\varphi$, the channel response $h_{i}$ is directly dependent on the transmitter location.

To model the change of amplitude with respect to the transmitter location, we use free space path-loss model and add a reflection decaying factor to account for the additional power loss during reflection. Note that $a_{p}(\varphi)$ represents the attenuation in the electrical field of the receiving signal. Therefore, $a_{p}(\varphi)$ can be expressed as

$$
a_{p}(\varphi)=\frac{r_{p}}{d_{p}(\varphi)}
$$

where $d_{p}(\varphi)$ represents the $p^{\text {th }}$ path travelling distance and $r_{p}$ is the reflection delaying factor for the $p^{\text {th }}$ path. Obviously, there is no reflection delaying for direct path, i.e. $r_{0}=1$. Because in the free space path-loss model, the receiving power is inversely proportional to the distance square, the electrical magnitude is therefore inversely related to distance, as shown in (2).

The received signal $\tilde{h}_{i}$ for frequency $f_{i}$ given the trans- mitter location is modeled as a noisy complex value, i.e.

$$
\tilde{h}_{i}(\varphi)=h_{i}(\varphi)+w_{i}, \quad\left|w_{i}\right| \sim N\left(0, \sigma_{i}^{2}\right),
$$

where $w_{i}$ represents the noise and is assumed as complex Gaussian distributed, i.e., the magnitude of $w_{i}$ is zero mean Gaussian distribution and the phase of $w_{i}$ is uniform distributed.

Hence, the probability density function of a measurement $\tilde{h}_{i}$ given $\varphi$ as the location of transmitter is

$$
p\left(\tilde{h}_{i} ; \varphi\right)=\frac{1}{\sqrt{2 \pi \sigma_{i}^{2}}} \exp \left\{-\frac{1}{2 \sigma_{i}^{2}}\left(\tilde{h}_{i}-h_{i}(\varphi)\right)\left(\tilde{h}_{i}-h_{i}(\varphi)\right)^{*}\right\} .
$$

\section{LOCATION AMBIGUITY ISSUE}

Due to the constructive and deconstructive interference of multipath signals, the likelihood function exhibits multiple local maximums with respect to different transmitter locations. Therefore, MLE based localization approach is very sensitive to noise, as likelihood magnitudes of several local maximums are quite close to each other for a single frequency, leading to multiple unresolvable candidate transmitter locations, i.e., location ambiguities.

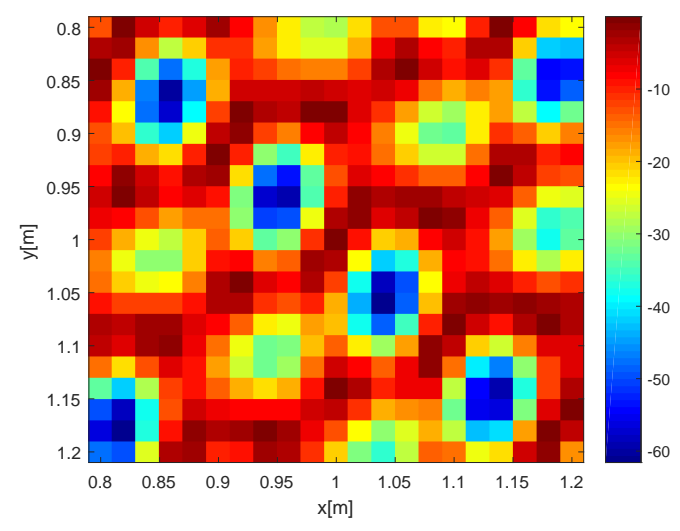

Figure 2: Likelihood map demonstrating ambiguous transmitter locations when a single frequency is used

To illustrate this, consider the scenario in Fig. 1 where the true transmitter's location is at $(1,1)$ and the likelihood value is evaluated around its true location, with a step size of 2 centimeters. As (it) can be seen in Fig. 2, multiple locations have very close likelihood value compared with the true location, leading to unresolvable ambiguities.

In order to tackle the ambiguity issue, we leverage the fact that the WiFi signals span over multiple frequency bands and naturally exploit the diversity of the receiving signal for different frequencies. The key idea is that for each individual frequency, even though its corresponding ambitious location during likelihood evaluation varies, the true location shall always contributes to a local maximum. Therefore, by combining multiple frequency measurements, transmitter location ambiguity can be possibly removed. See Fig. 3 for an explanation. It can be seen that the signal to noise ratio is dramatically improved compared to the single frequency result in Fig. 2. Hence location ambiguity could be greatly 
mitigated by exploiting WiFi signal at multiple frequency bands.

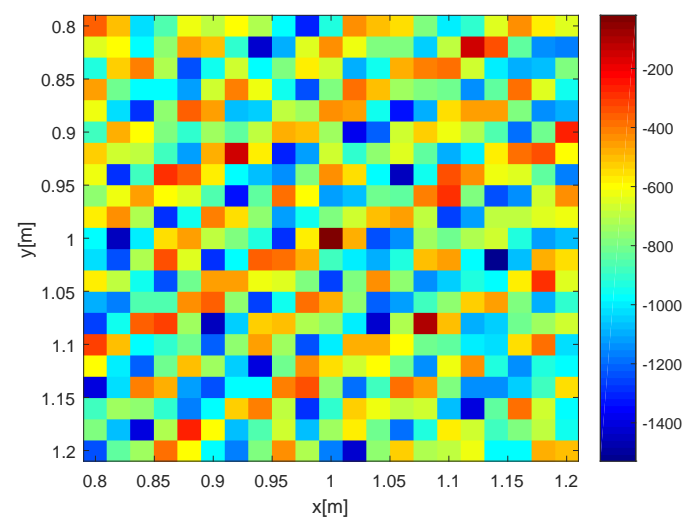

Figure 3: Remove transmitter location ambiguity by using multiple frequencies: as shown by the likelihood map, the true location at $(1,1)$ contributes to the largest likelihood compared to its neighbouring locations.

To further evaluate the ability of ambiguity suppressing by applying multiple WiFi frequency signal, we calculate the Fisher information matrix $I(\varphi)$ as [6]

$$
[I(\varphi)]_{m, n}=-E\left[\sum_{i=1}^{N} \frac{\partial^{2}}{\partial \varphi_{m} \partial \varphi_{n}} \log p\left(\tilde{h}_{i} ; \varphi\right)\right]
$$

where $N$ is the total number of frequencies and $E[\cdot]$ represents expectation operator with respect to the measurements.

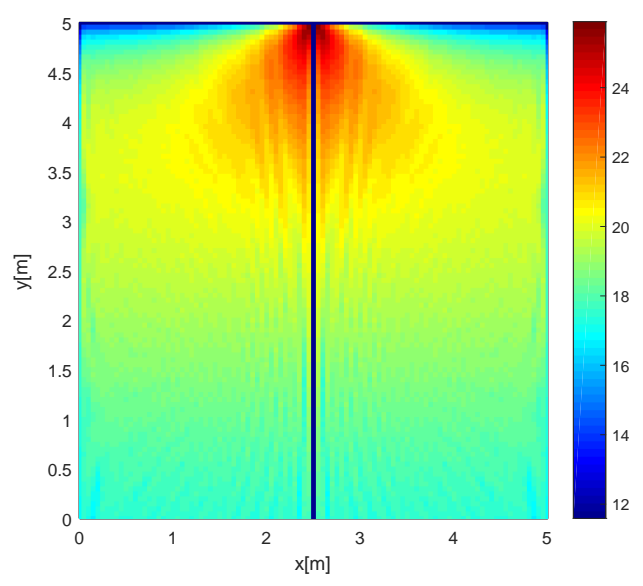

Figure 4: Determinant of Fisher information matrix using 35 frequencies.

To evaluate how much information we can get by applying MLE, we compute $I(\varphi)$ with respect to all possible transmitter locations using (5) and take the corresponding determinant. Fig. 4 demonstrates the determinant value of Fisher information matrix when the receiver is placed at $(2.5,5)$. In this special case, Fisher information exhibits symmetry property as the receiver is positioned in symmetry with the room structure. No information can be acquired along the vertical line at $x=2.5 \mathrm{~m}$ as Fisher information matrix is singular.

Remarks:

- Although it is evident employing multiple frequencies can help remove location ambiguity, the minimum number of the required frequency along with how to select them among the available frequency bands in an optimal sense remains to be an ongoing research. Obviously, the more frequency employed, the less ambiguity can be achieved. Based on simulation result, frequencies selected across $2.4 \mathrm{GHz}$ band and $5 \mathrm{GHz}$ band can provide better ambiguity suppressing performance than those selected from either the $2.4 \mathrm{GHz}$ band or the $5 \mathrm{GHz}$ band only.

- Due to the fact that high frequency WiFi signal has a short wavelength (centimeter level), the likelihood function changes over distance on the order of several centimeters. Therefore how to identify the global maximum can be very challenging in practice. Similar problem has also been mentioned in [7] using MLE for parameter estimation. We propose to meet the computation requirement for global maximum identification in combination with a subspace approach for time delay offset estimation, using the same set of measurements, which will be discussed in the Section IV.

\section{TRANSMITTER LOCALIZATION}

In this section, we turn to the practical procedures on how to employ MLE to find the radio transmitter location. First the maximum likelihood function is reformulated with respect to multiple $\mathrm{WiFi}$ frequency and multiple samples for each frequency. Second, in order to reduce the computation time, a subspace based approach is used in combination with MLE to improve computation efficiency.

\subsection{Maximum Likelihood Approach}

As discussed above, transmitter location can't be identified by MLE with a single frequency due to ambiguity. Hence, we utilize multiple frequencies in CSI and formulate the location problem as follows: denote the $k^{\text {th }}$ measurement set of all the available $N$ frequencies as $\tilde{\boldsymbol{h}}_{k}=$ $\left[\tilde{h}_{1 k}, \tilde{h}_{2 k}, \cdots, \tilde{h}_{N k}\right]^{T}$, whose distribution is modeled as joint Gaussian

$$
p(\tilde{\boldsymbol{h}} ; \varphi))=\frac{\exp \left\{-\frac{1}{2}\left(\tilde{\boldsymbol{h}}_{k}-\boldsymbol{h}_{k}(\varphi)\right)^{*} \boldsymbol{\Sigma}_{k}^{-1}\left(\tilde{\boldsymbol{h}}_{k}-\boldsymbol{h}_{k}(\varphi)\right)\right\}}{(2 \pi)^{\frac{N}{2}}\left|\boldsymbol{\Sigma}_{k}\right|^{\frac{1}{2}}}
$$

where $(\cdot)^{T}$ and $(\cdot)^{*}$ represents transpose and Hermitian conjugate transpose respectively, and $\Sigma_{k}=\sigma^{2} I_{N}$ is the covariance matrix of noise.

In practice, for all the available frequencies, we might be able to take multiple measurement samples. Assume $K$ samples are taken for each frequency. Then all the measurements can be stacked as a $N \times K$ measurement matrix $\tilde{\boldsymbol{H}}=\left[\tilde{\boldsymbol{h}}_{1}, \tilde{\boldsymbol{h}}_{2}, \cdots, \tilde{\boldsymbol{h}}_{K}\right]$. Therefore, drop the constant value, the log likelihood of $\tilde{\boldsymbol{H}}$ can be written as

$$
\log p(\tilde{\boldsymbol{H}} ; \varphi)=\sum_{k=1}^{K}-\frac{1}{2}\left(\tilde{\boldsymbol{H}}_{k}-\boldsymbol{H}_{k}(\varphi)\right)^{*} \boldsymbol{\Sigma}_{k}^{-1}\left(\tilde{\boldsymbol{H}}_{k}-\boldsymbol{H}_{k}(\varphi)\right)
$$


The MLE criterion is to find a location point $\varphi=(x, y)$ that leads to the largest likelihood value. It is worth noting that under the Gaussian white noise assumption, MLE is equivalent to a nonlinear least-square error minimization problem.

\subsection{Path Delay Offset Estimation}

To find the highest peak in the global sense can be extremely time consuming in general, since the distance between any two closest local maximums is in the order of half the wavelength, which is about six centimeters for $2.4 \mathrm{GHz}$ WiFi signal. The magnitude of likelihood function varies rapidly with respect to the transmitter location due to the interference between multipath $\mathrm{WiFi}$ signal components, which have relatively short wavelengths. In other words, a slight change of the transmitter location can make a significant change in the corresponding receiving signal. This will pose a challenge for the MLE global maximum identification.

To improve computation efficiency, we further utilize CSI and exploit time delay information contained therein. We rewrite the $\mathrm{N}$ frequency channel response in (3) by a matrix form,

$$
\left[\begin{array}{c}
\tilde{h}_{1} \\
\tilde{h}_{2} \\
\vdots \\
\tilde{h}_{N}
\end{array}\right]=\left[\begin{array}{llll}
F\left(\tau_{1}\right) & F\left(\tau_{2}\right) & \cdots & F\left(\tau_{P}\right)
\end{array}\right]\left[\begin{array}{c}
a_{1} \\
a_{2} \\
\vdots \\
a_{P}
\end{array}\right]+\left[\begin{array}{c}
w_{1} \\
w_{2} \\
\vdots \\
w_{N}
\end{array}\right]
$$

with $F\left(\tau_{p}\right)=\left[\exp ^{-j 2 \pi f_{1} \tau_{p}}, \exp ^{-j 2 \pi f_{2} \tau_{p}}, \cdots, \exp ^{-j 2 \pi f_{N} \tau_{p}}\right]^{T}$.

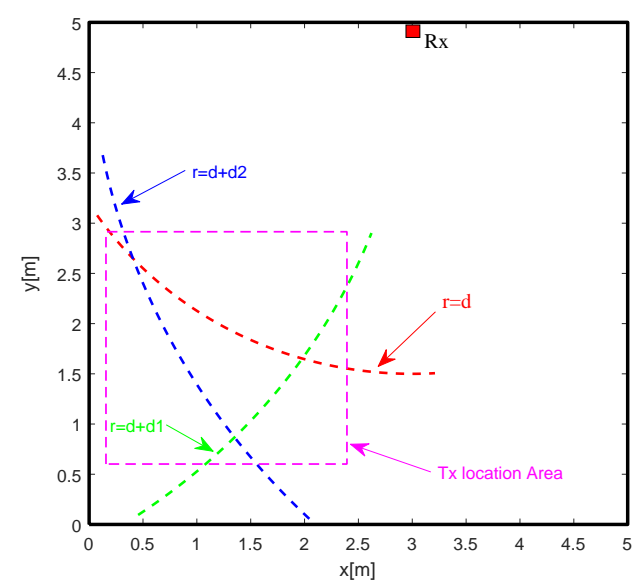

Figure 5: Transmitter location area estimation by using relative time delay offset: the transmitter's location area is decided by the intersection region of three ray path dependent circles. $r$ is circle radius. $d_{1}=c *\left(\tilde{\tau}_{1}-\tilde{\tau}_{0}\right)$, and $d_{2}=c *\left(\tilde{\tau}_{2}-\tilde{\tau}_{0}\right)$, where $\tilde{\tau}_{p}$ represents the estimated time delay for the $p^{\text {th }}$ path, $d$ represents the unknown distance between transmitter and receiver, and $c$ is the light speed.

Therefore, $F\left(\tau_{p}\right)$ can be regarded as a steering vector containing the unknown parameter of the pth path time delay $\tau_{p}$. Without resorting to any other hardware or precise measurements, a subspace based approach such as [8],[9] can be employed for path delay estimation using the same
WiFi measurements, with which a transmitter location region can be roughly provided. In this scenario, we don't assume synchronization between transmitter and receiver, which is difficult in practical application. Moreover, there exists time offset in signal detection at the physical layer. Therefore, even though subspace approach can be used for propagation time estimation, the path delay can't be accurately acquired. However, the delay offset between any two paths can be exploited for distance difference estimation. As illustrated in Fig. 5, together with the prior knowledge of the room structure, we can make use of triangulation technique to either estimate the transmitter location in the minimum square error criterion or roughly estimate transmitter location region. The triangulation technique is expressed by the following equation set

$$
\left\{\begin{array}{l}
\left(x-x_{0}\right)^{2}+\left(y-y_{0}\right)^{2}=d^{2} \\
\left(x-x_{1}\right)^{2}+\left(y-y_{1}\right)^{2}=(d+d 1)^{2} \\
\left(x-x_{2}\right)^{2}+\left(y-y_{2}\right)^{2}=(d+d 2)^{2}
\end{array}\right.
$$

where $\left(x_{0}, y_{0}\right)$ is the receiver coordinate and $\left(x_{1}, y_{1}\right),\left(x_{2}, y_{2}\right)$ represent coordinates of imaginary receivers for reflected path signals that are received by the true receiver.

Based on the above discussion, we propose a combined approach to fuse the results of both the MLE and the subspace method using the same measurement set. A two step procedure can be conducted practically. First, a subspace based method is utilized for transmitter location area estimation, followed by maximum likelihood peak searching in this confined area based on MLE at the second stage. This can help avoid searching for global maximum across the whole region, hence computation time can be reduced.

\section{SIMULATION}

In this simulation, we consider a two dimensional space with known structure and three main path signal components in the receiving signal. As shown in Fig. 6, the receiver's location is given as $(3,5)$. A transmitter grid set with a large number of transmitters spaced by 5 centimeters between each other is placed to evaluate how the signal changes with location. For the sake of clear illustration, we randomly pick up one of the transmitter among the grid and demonstrate its three main signal propagation paths that are considered in the model.

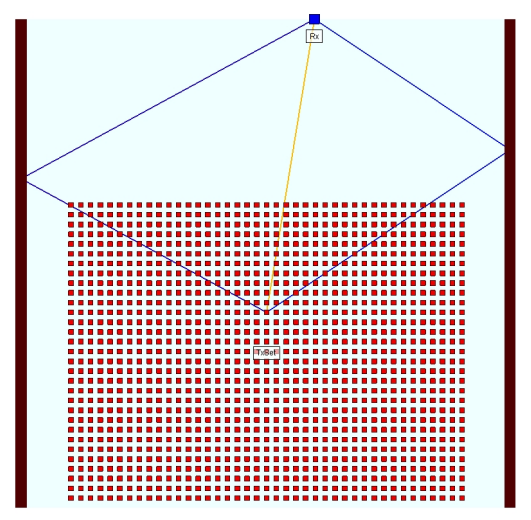

Figure 6: Scenario Setting using ray tracing software 
The ray tracing software [10] can provide the receiving signal power (in $\mathrm{dBm}$ ) and phase (in degree) with respect to different transmitter location. To simulate CSI data, a postprocessing of the software output is needed to convert the receiving signal into the complex form as shown in (1). This can be done either by making use of the complex impulse response output or the receiving power output. Then a Gaussian complex noise is added to each measurement to account for the measurement uncertainty in CSI.

In order to evaluate the effectiveness of the MLE approach for radio transmitter location estimation, we randomly pick up a transmitter (location coordinate is $(1.53,0.96)$ ) in the transmitter grid set in Fig. 6 and extract its corresponding signal measurements of all the available frequencies for MLE based localization.

When we make use of all the available 35 frequencies spanning from $2.4 \mathrm{GHz}$ to $5 \mathrm{GHz}$ band, ambiguity can be removed and the true target location can be identified. Fig. 7 shows the result for one simulation. As the subspace method can provide with transmitter's location area (rectangular drew by dashed line), efficiency can be improved during the MLE procedure as multiple local maximum location outside of this location area can be avoided for likelihood evaluation. This demonstrates that the MLE is able to find the global maximum and hence localize the transmitter.

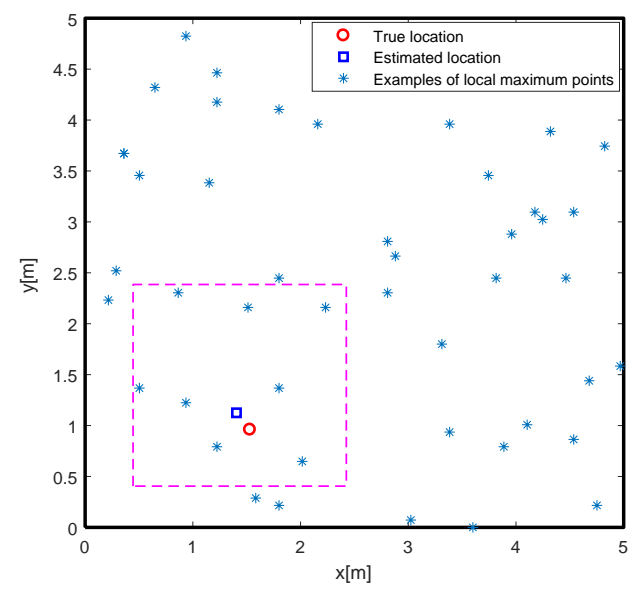

Figure 7: Simulation result using 35 frequencies

Please note that the subspace method can only help to narrow down the searching area for the global maximum point in MLE estimation. To account for uncertainty and avoid risky decision making for transmitter location, a relatively large searching area needs to be defined. So there is a tradeoff in practice. A more sophisticated approach should be developed for global maximum identification in the future.

\section{CONCLUSION}

The WiFi based radio transmitter localization using maximum likelihood approach contributes in the following ways. First, instead of using multiple receivers, only one receiver is employed for transmitter location estimation, which is more practical in today's mobile application and is immune from multiple receivers deployment difficulty. Second, we naturally exploit the CSI which is available in commercial chips and make use of multiple WiFi frequencies to remove location ambiguity. Third, by making use of the same set of measurements, a subspace based method is utilized for transmitter location area estimation, which can be combined with MLE to improve localization efficiently. Future work will focus on radio transmitter localization in the unknown and time variant environment.

\section{ACKNOWLEDGMENTS}

This work is partially supported by Australia Research Council Linkage project grant LP150100065 and discovery project grant DP120102575.

\section{References}

[1] Hui Liu, Houshang Darabi, Pat Banerjee, and Jing Liu. Survey of wireless indoor positioning techniques and systems. IEEE Transactions on Systems, Man, and Cybernetics, Part $C$ (Applications and Reviews), 37(6):1067-1080, 2007.

[2] Andreas F Molisch. Wireless communications, volume 34. John Wiley \& Sons, 2012.

[3] Deepak Vasisht, Swarun Kumar, and Dina Katabi. Decimeter-level localization with a single wifi access point. In 13th USENIX Symposium on Networked Systems Design and Implementation (NSDI 16), pages 165-178. USENIX Association, 2016.

[4] Daniel Halperin, Wenjun Hu, Anmol Sheth, and David Wetherall. Tool release: Gathering $802.11 \mathrm{n}$ traces with channel state information. ACM SIGCOMM Computer Communication Review, 41(1):53-53, 2011.

[5] David Tse and Pramod Viswanath. Fundamentals of wireless communication. Cambridge university press, 2005.

[6] Bradley Efron and David V Hinkley. Assessing the accuracy of the maximum likelihood estimator: Observed versus expected fisher information. Biometrika, 65(3):457-483, 1978.

[7] J. J. Fuchs. Detection and estimation of superimposed signals. In Acoustics, Speech and Signal Processing, 1998. Proceedings of the 1998 IEEE International Conference on, volume 3, pages 1649-1652 vol.3, May 1998.

[8] Ralph Schmidt. Multiple emitter location and signal parameter estimation. IEEE transactions on antennas and propagation, 34(3):276-280, 1986.

[9] Manikanta Kotaru, Kiran Joshi, Dinesh Bharadia, and Sachin Katti. Spotfi: Decimeter level localization using wifi. In ACM SIGCOMM Computer Communication Review, volume 45, pages 269-282. ACM, 2015.

[10] Nidal Qasem and Rob Seager. Studies on enhancing wireless signal for indoor propagation. In Antennas and Propagation Conference (LAPC), 2010 Loughborough, pages 309-312. IEEE, 2010. 